\section{The Diploma in Sports Medicine of The Society of Apothecaries of London}

The Court of the Society of Apothecaries of London have resolved to institute an examination and award a diploma in sports medicine which is open to registered medical practitioners who have worked in the field of sports medicine or have had other definite experience and attended a recognized course of instruction. The first examination is to be held in June 1989.

The training and examination is aimed at those practitioners involved in the management and treatment of injuries and other medical problems arising from sport to a degree unusual in normal medical practice. The more obvious examples are medical officers engaged by or attached to clubs such as athletics, boxing, football, hockey, swimming, etc.

The Society is a Craft Guild founded by a Royal Charter granted by King James I in 1617 and is now one of the three non-university licensing boards in the

\section{Regulations for the admission to the examination for the Diploma in Sports Medicine of The Society of Apothecaries (DSMSA)}

1 Candidates must be registered with the General Medical Council in the United Kingdom and have possessed a qualification for at least four years to practise medicine, surgery and obstetrics and gynaecology. Graduates in medicine, surgery and obstetrics and gynaecology of the British Commonwealth, countries in the European Economic Community or foreign universities, who are not registered with the GMC to practise in the United Kingdom, may be admitted to the examination by the approval of the Court of Examiners if they have complied with all other requirements of the Regulations.

2 Candidates must produce satisfactory evidence that after qualification they have:

(a) Attended a full-time course for at least 20 weeks in sports medicine (Appendix I) recognized by the Court of Examiners and gained experience in sports medicine

or

(b) Attended a recognized equivalent part-time course

or

(c) Attended three full-time courses of one week each in sports medicine and practised sports medicine for at least 2 years in work approved by the Examiners.

or

(d) Practised sports medicine in approved appointments for at least 4 years

(C) 1989 Butterworth \& Co (Publishers) Ltd 0306-3674/89/010061-02 \$03.00

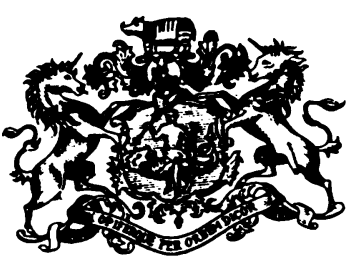

United Kingdom recognized by the General Medical Council. During its long history, the Society has instituted other examinations including the first postgraduate diploma in midwifery, the first in industrial health and currently awards diplomas in medical jurisprudence, genito-urinary medicine and the history and philosophy of medicine.

Candidates for the new diploma will have to satisfy the Court of Examiners as to their suitability to enter. Courses to be recognized are currently being conducted by:

The London Hospital-full time

The London Sports Medicine Institute-part time British Association of Sport and Medicine-part time.

The regulations and syllabus for the examination may be obtained from The Registrar, Society of Apothecaries, Blackfriars Lane, London EC4V 6EJ.

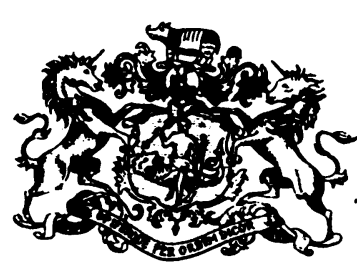

or

(e) Have a degree from a university in the United Kingdom, the examination or thesis for which was primarily concerned with sports medicine.

3 Dissertation: Candidates should submit a dissertation in type script on a subject related to sports medicine at the time they apply to sit the examination. The title of the dissertation should be submitted to the examiners before the dissertation is written and application for approval should be made to the Registrar, Worshipful Society of Apothecaries, Apothecaries Hall, Blackfriars Lane, London EC4V 6EJ. The dissertation itself should comprise not less than 3000 words and not more than 5000 words. Although original observations and research are encouraged, a dissertation consisting of a review of current literature or attitudes would be acceptable.

4 Case History Books: Candidates are required to submit detailed case histories in type script of not less than six patients who have been under their care. This should be submitted with the dissertation. It should include presentation, diagnosis, treatment and outcome, together with a general appraisal and critical approach.

5 The examination will consist of a multiple choice question paper, a written paper, a clinical section and an oral section.

6 The fee for the examination is $£ 200$. 


\section{Syllabus for Diploma in Sports \\ Medicine of The Society of Apothecaries of London}

The syllabus gives a guide to the examinees but all aspects of sports medicine and illness in relation to sport may be examined. (Some aspects may be covered under more than one heading in the list below.)

1 Ethical and social aspects of sports medicine Socioeconomic aspects of sport and exercise. Legal aspects of sports injury and treatment. Epidemiology of sports injuries.

2 Anatomy Body composition and its measurement. Somatotypes in different sports. Biomechanics and symmetry. Effect of sport and exercise on growth and development. The musculoskeletel system.

3 Exercise physiology and biochemistry Muscle function fibre type, strength and endurance. Muscular hypertrophy. Fuel stores and muscular endurance. Aerobic and anaerobic exercise. Cardiovascular, respiratory, endocrine and haemopoietic systems and exercise. Oxygen transport. Thermoregulation.

Exercise testing Strength, aerobic and anaerobic testing. Ergometers. Lactate measurement and relevance to training regimes.

Effects of prolonged conditioning on body systems The elite athlete. Athlete's heart. Athlete's anaemia. Limits to performance.

4 Training techniques for different sports and their physiological basis The role of the coach. Skill training. Peaking for competition.

5 Nutrition in sport Water and electrolyte balance. $\mathrm{Nu}$ tritional boosting. Making weight.

6 Psychological aspects of competition and training Perceived exertion. Mental rehearsal. Self image and performance. Performance slumps and "overtraining syndromes". Exercise dependence.

7 Environmental physiology and dangers Acclimatization to and effects of heat, humidity, cold, altitude and hyperbaric (diving) environments. Heat syndromes. Hypothermia and frost bite. Altitude sickness and syndromes. Compression and decompression syndromes. Equipment and clothing for changed environments. Body clocks, travel and performance.

8 Problems of special groups Women in sport. Gender verification. Amenorrhoea and osteoporosis. Gynaecological problems. Children and adolescents in sport. The dominant coach or parent. The middle-aged and elderly participant. Sport for the disabled. Sport and the diabetic.

9 Sports injuries Injuries related to different sports. Acute trauma. Overuse injuries. Fatigue fractures. Causes of injury, diagnostic modalities, treatment and rehabilitation. Cramp and muscle stiffness.

Treatment modalities. Inflammation, repair and healing. Physiotherapy treatments and rationale. Role of physiotherapist. Strapping, massage and analgesia. When to refer.

10 Team or event doctor Responsibilities. Intercurrent illnesses and infections (including STD). Fitness to compete. Immunizations and special preparation for foreign competition. Exercise induced asthma. Travellers' diarrhoea. Skin problems. Precompetition nerves. Role of team officials and medical staff. First aid and other equipment on tour. Resuscitation techniques. Organization of medical support for mass participation events. Mountain rescue. Sea rescue. Winter sports.

11 Clinical pharmacology of sports medicine Use and abuse of drugs in sport. Banned drugs and ergonomic aids. Blood doping. Management of athletes problems without using banned substances. Anabolic steroids. Drug testing.

12 Sports equipment Shoes, surfaces, equipment.

13 Sport and exercise as therapy Benefits of exercise. Exercise and coronary disease. Sudden death in sport. Exercise and longevity. Prescribing exercise to the unathletic. Exercise testing as a screening procedure. 